\title{
Crystal Structure and Potential Head-to-Middle Condensation Function of a Z,Z-Farnesyl Diphosphate Synthase
}

\author{
Yueh-Te Chan, ${ }^{\dagger}$ Tzu-Ping Ko, ${ }^{\dagger}$ Shan-Hsueh Yao, ${ }^{\dagger}$ Ya-Wen Chen, ${ }^{\dagger}$ Cheng-Chung Lee, ${ }^{*}{ }^{\dagger}$ \\ and Andrew H.-J. Wang ${ }^{*},+,+, \S$ \\ ${ }^{\dagger}$ Institute of Biological Chemistry, Academia Sinica, Taipei 115, Taiwan \\ ${ }^{\ddagger}$ Graduate Institute of Translational Medicine, College of Medical Science and Technology, Taipei Medical University, Taipei 110, \\ Taiwan \\ ${ }^{\S}$ Institute of Biochemical Sciences, National Taiwan University, Taipei 106, Taiwan
}

Supporting Information

ABSTRACT: Plants produce a wide variety of secondary metabolites in response to adverse environmental factors. Z,Z-Farnesyl diphosphate (Z,Z-FPP), synthesized by Z,Z-farnesyl diphosphate synthase ( $z$ FPS), supports the formation of phytochemicals in wild tomatoes. Here, the crystal structure of $\mathrm{N}$-terminal truncated $z \mathrm{FPS}(\Delta z \mathrm{FPS})$ was determined. Irregular products including lavandulyl diphosphate and an unknown compound were surprisingly found. Apart from the truncated $\mathrm{N}$ terminus as a functional regulator, structure-based analysis and mutagenesis assays revealed a residue $\mathrm{H} 103$ in $\Delta z \mathrm{FPS}$ as one of the key elements to this irregular function. A series of substrate-enzyme complex structures were obtained from $\Delta z$ FPS-H103Y by co-crystallizing with isopentenyl diphosphate, dimethylallyl thiolodiphosphate, or both. Various substrate-binding modes were revealed. The catalytic mechanisms of both the head-to-tail and head-to-middle reactions in $\Delta z$ FPS were proposed. Functional switch between the two mechanisms in this enzyme and the essential role played by the flexible Cterminus were elucidated as well.

\section{INTRODUCTION}

Terpenoids are among the most important metabolites in plants. They play various roles, such as mediating defense or beneficial interactions. ${ }^{1-6}$ Prenyltransferases (PTSs), which are also known as isoprenyl diphosphate synthases, catalyze the formation of the upstream substrates for terpene synthases (TPSs). ${ }^{3}$ The reaction begins with dimethylallyl diphosphate (DMAPP) and isopentenyl diphosphate (IPP), which are synthesized from acetyl-CoA via the mevalonate pathway in most animal and fungal cells or from carbohydrate derivatives via the methylerythritol phosphate pathway in bacteria and plant chloroplasts. $^{6-9}$ PTSs catalyze consecutive prenyl chain elongation via nucleophilic attack to the initiatory substrate, resulting in trans $(\mathrm{E})$ or cis $(\mathrm{Z})$ products, ${ }^{10,11}$ which differ in their molecular geometry. Regarding the three-dimensional folds of the enzymes, the $\alpha$-helical bundle is generally adopted by trans-PTS. ${ }^{12,13}$ For cis-PTS, a Rossmannlike fold is presented, ${ }^{14,15}$ in which the dimeric enzyme takes the shape of a butterfly composed of a pairing domain and a catalytic domain.

The terpenoids identified to date are mostly derived from the trans-type substrates. Knowledge about cis-PTS is rather limited. The $z$ FPS protein in this study is a short-chain cisPTS from the glandular trichomes of the tomato species
Solanum habrochaites. Its major product, Z,Z-FPP, is an important precursor for the formation of phytotoxins with vital defense roles in wild tomato. ${ }^{16,17}$ The crystal structure of $z$ FPS, lacking the transit peptide and an additional 27 residues on the N-terminus, was determined, named $\Delta z$ FPS. To obtain substrate-enzyme complex structures, the $\Delta z$ FPS-H103Y mutant was designed by structure-based sequence alignment with other homologous enzymes. From this mutant, a series of substrate-enzyme complex structures were obtained and the irregular head-to-middle function was also revealed. Both the head-to-tail and head-to-middle condensation reactions are elucidated from the complex structures. The results provide new insights into the study of the highly diverse cis-PTS family.

\section{RESULTS AND DISCUSSION}

The full-length enzyme includes an N-terminal chloroplasttargeting peptide predicted to be 45 amino acids in length. ${ }^{16}$ The N-terminal 45-residue truncated clone, which was expressed and purified, was named $z$ FPS directly. Because no crystal was obtained from this clone, an additional N-terminal

Received: December 29, 2016

Accepted: February 28, 2017

Published: March 16, 2017 
Table 1. Data Collection and Refinement Statistics ${ }^{a, b}$

\begin{tabular}{|c|c|c|c|c|c|}
\hline name/PDB code & $\Delta z$ FPS-APO/5HXN & $\Delta z$ FPS-M/5HXO & $\Delta z$ FPS-MI/5HXP & $\Delta z$ FPS-MD/5HXQ & $\Delta z$ FPS-MDI/5HXT \\
\hline \multicolumn{6}{|l|}{ data collection } \\
\hline space group & $P 4_{3} 2_{1} 2$ & $P 4_{3} 2_{1} 2$ & $P 2_{1} 2_{1} 2_{1}$ & $P 2_{1} 2_{1} 2_{1}$ & $P 2_{1} 2_{1} 2_{1}$ \\
\hline \multirow[t]{3}{*}{ cell dimensions $(\AA)$} & $a=51.89$ & $a=51.68$ & $a=58.31$ & $a=59.75$ & $a=59.98$ \\
\hline & $b=51.89$ & $b=51.68$ & $b=64.55$ & $b=66.80$ & $b=66.32$ \\
\hline & $c=173.29$ & $c=173.56$ & $c=127.16$ & $c=128.73$ & $c=128.9$ \\
\hline \multirow[t]{2}{*}{ resolution $(\AA)$} & $25.0-2.05$ & $25.0-2.05$ & $25.0-1.95$ & $25.0-1.95$ & $20.0-2.15$ \\
\hline & $(2.12-2.05)$ & $(2.12-2.05)$ & $(2.02-1.95)$ & $(2.02-1.95)$ & $(2.23-2.15)$ \\
\hline unique reflections & 15386 & 15682 & 35453 & 38064 & 28515 \\
\hline$R_{\text {merge }}(\%)$ & $4.4(49.5)$ & $4.3(41.2)$ & $4.1(54.5)$ & $4.3(76.4)$ & $5.5(47.7)$ \\
\hline$I / \sigma(I)$ & $52.3(4.3)$ & $42.8(5.1)$ & $33.0(3.0)$ & $42.4(2.8)$ & $37.4(3.9)$ \\
\hline completeness & $97.4(94.6)$ & $99.9(99.9)$ & $99.7(100.0)$ & $99.3(99.8)$ & $99.8(98.5)$ \\
\hline redundancy & $11.0(10.0)$ & $10.6(9.6)$ & $4.0(4.1)$ & $5.9(5.7)$ & $7.1(6.5)$ \\
\hline \multicolumn{6}{|l|}{ refinement } \\
\hline resolution $(\AA)$ & $25.0-2.05$ & $25.0-2.05$ & $25.0-1.95$ & $25.0-1.95$ & $20.0-2.15$ \\
\hline no. of reflections & $14337 / 751$ & $14812 / 777$ & $31558 / 1761$ & $31779 / 1679$ & $25353 / 1286$ \\
\hline$R_{\text {work }} / R_{\text {free }}$ & $19.2 / 24.1$ & $19.1 / 23.7$ & $19.9 / 24.3$ & $19.4 / 24.1$ & $19.7 / 24.9$ \\
\hline \multicolumn{6}{|c|}{ no. of nonhydrogen atoms/avg B factor $\left(\AA^{2}\right)$} \\
\hline protein & $1771 / 27.9$ & $1755 / 27.5$ & $3587 / 23.1$ & $3643 / 30.2$ & $3602 / 28.6$ \\
\hline water & $154 / 38.8$ & $118 / 35.5$ & $204 / 32.3$ & $315 / 38.7$ & $320 / 39.3$ \\
\hline ligand & & & $42 / 36.0$ & $14 / 34.6$ & $56 / 39.7$ \\
\hline $\mathrm{Mg}^{2+}$; crown-ether & & & $1 / 28.1 ; 36 / 37.2$ & na; $36 / 58.5$ & $2 / 37.9 ; 18 / 52$ \\
\hline RMSD bond lengths $(\AA)$ & 0.007 & 0.007 & 0.009 & 0.008 & 0.007 \\
\hline RMSD bond angles (deg) & 1.16 & 1.21 & 1.43 & 1.28 & 1.18 \\
\hline \multicolumn{6}{|l|}{ Ramachandran plot (\%) } \\
\hline most favored & 94.9 & 94.9 & 94.2 & 95.8 & 94.8 \\
\hline additionally allowed & 4.6 & 5.1 & 5.5 & 4.2 & 5.0 \\
\hline generously allowed & 0.0 & 0.0 & 0.0 & 0.0 & 0.2 \\
\hline disallowed & 0.5 & 0.0 & 0.3 & 0.0 & 0.0 \\
\hline
\end{tabular}

${ }^{a}$ Values corresponding to the highest-resolution shells are shown in parentheses. ${ }^{b}$ The stereochemistry of the model was validated with PROCHECK.

27-residue truncation was made and named $\Delta z$ FPS, which was successfully crystallized. This apo-form $\Delta z$ FPS crystal ( $\Delta z$ FPSAPO) belonged to the $P_{4} 2_{1} 2$ space group, with one protein molecule in an asymmetric unit (Table 1; see crystallographic information files in the Supporting Information). Similar to the structures of other cis-PTSs, the overall fold of $\Delta z$ FPS is Rossmannlike (Figure 1A). Two dyad-related monomers are associated into a dimer via hydrogen bonds and hydrophobic interactions. This dimeric structure is consistent with the results of the sedimentation velocity (SV) analysis (Figure S1). However, we were unable to obtain the complex structure after testing a series of substrates or substrate analogues, including IPP, ISPP (isopentenyl S-thiolodiphosphate), DMSPP, GPP (geranyl diphosphate), and ibandronate.

To obtain complex structures, catalytic residues around the active site were examined. Four catalytic residues including $\mathrm{N} 134, \mathrm{H} 103, \mathrm{~S} 131$, and R137 of $\Delta z \mathrm{FPS}$, corresponding to N74, H43, S71, and R77 from an undecaprenyl diphosphate synthase (UPPS), ${ }^{18}$ potentially serve as general bases or acids for the chemical reaction. These catalytic residues are highly conserved in the cis-type PTS family, except the position $\Delta z$ FPS-H103. Indeed, this positon is frequently replaced by Tyr in other homologous enzymes (Figure S2). Using the $\Delta z \mathrm{FPS}-\mathrm{H} 103 \mathrm{Y}$ mutant, complex structures were successfully obtained (see below) and the enzyme function was altered as well. Irregular products including LPP (Peak-4), an unidentified Peak-3 compound, and the monoterpene limonene (Peak-5) were found, with the native function retained (Figures 2 and S4). On the basis of the NIST Standard Database, the most reasonable candidate for Peak-3 is the monoterpene "menthol", which showed the same retention time as that for Peak-3; however, the similarity of the mass spectra is too low (around 30\%). The Peak-5 limonene is a cyclized terpenoid compound.

Most TPS structures share a common $\alpha$-helical TPS fold. ${ }^{19,20}$ However, di-TPS Rv3378c from Mycobacterium tuberculosis was found to adopt the same Rossmannlike fold as that by cisPTS. $^{21}$ Cyclization function in cis-PTS is rare, but it was reported in a cyclolavandulyl diphosphate synthase ${ }^{22}$ (CLDPS). A structure-based sequence comparison revealed the conserved character among the same corresponding positions Rv3378Y51, CLDPS-Y26, and Y103 from $\Delta z$ FPS-H103Y. On the other hand, the Peak-4 LPP is an irregular prenyl diphosphate, synthesized by the head-to-middle condensation reaction from DMAPP molecules. ${ }^{22,23}$ We supposed that a similar irregular reaction is adopted for the formation of unknown Peak-3. Unlike $\Delta z$ FPS-H103Y, the two irregular products were significantly reduced in $\Delta z$ FPS when IPP was provided. However, without IPP, $\Delta z$ FPS produced the two irregular products dominantly (Figure 2 ).

Three complex structures were successfully determined, including $\Delta z$ FPS-MI $\left(\Delta z\right.$ FPS-H103Y $\left./ \mathrm{Mg}^{2+} / \mathrm{IPP}\right), \Delta z$ FPS MDI $\left(\Delta z\right.$ FPS-H103Y/DMSPP $\left./ \mathrm{Mg}^{2+} / \mathrm{IPP}\right)$, and $\Delta z$ FPS-MD ( $\Delta z$ FPS-H103Y/DMSPP). All complex structures belonged to the $P 2_{1} 2_{1} 2_{1}$ space group, with one protein dimer per asymmetric unit and crown-ether molecule(s) bound to the surface lysine (Figures $1 \mathrm{~A}$ and S5A,B).

Two substrate-binding sites can be discerned: an S1 site for the binding of the initiatory substrate and an S2 site for the 
A

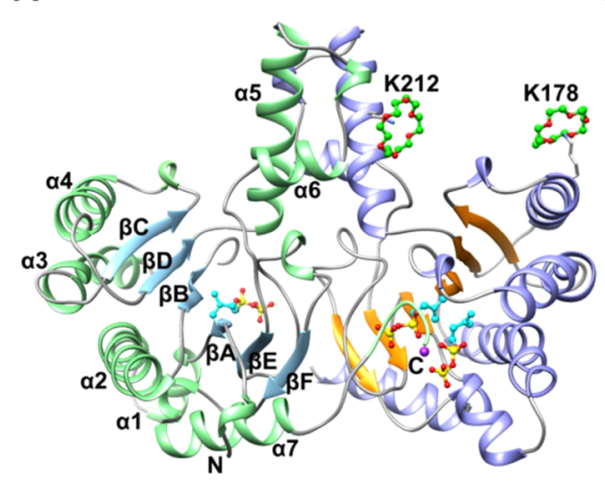

B

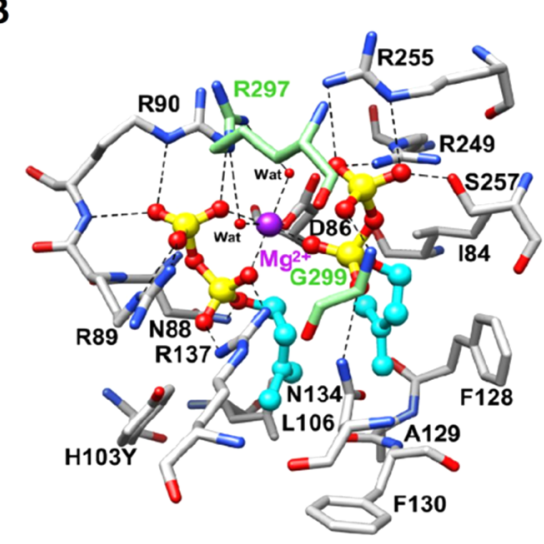

C

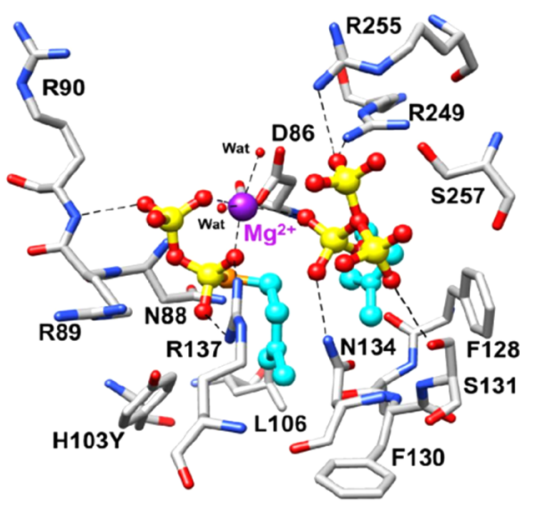

D

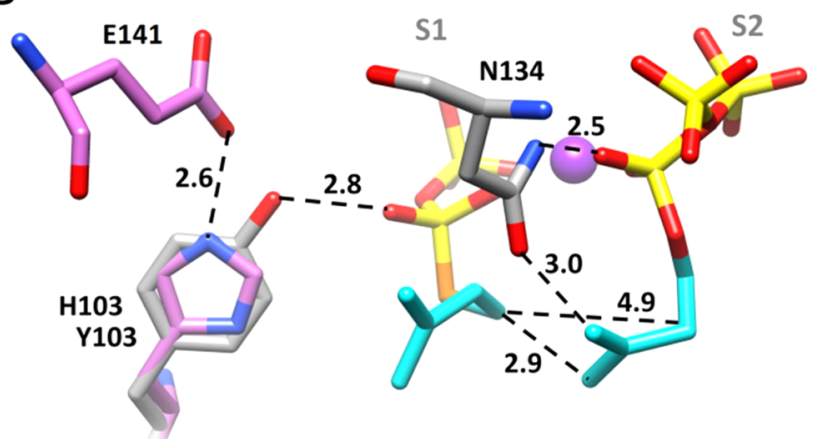

$\mathbf{E}$

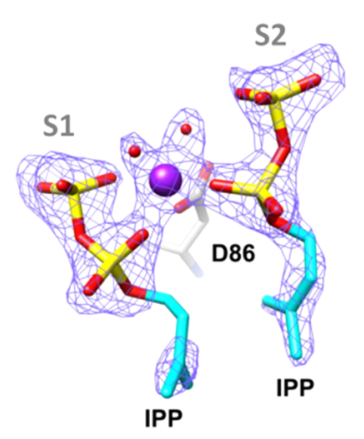

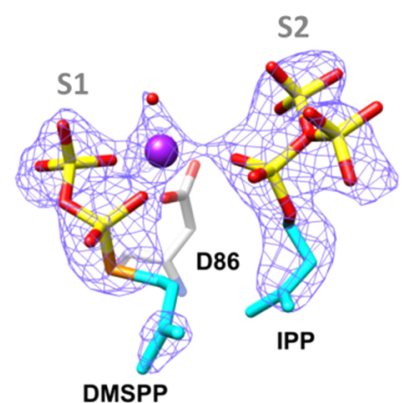

Figure 1. Substrate-enzyme complex structures of $\Delta z$ FPS-H103Y. (A) General structure of $\Delta z$ FPS-MI. The cyclic crown-ether molecules are shown as balls and sticks. (B, C) The side chains of the ligand-interacting residues of $\Delta z$ FPS-MI and $\Delta z$ FPS-MDI, respectively. The Mg ${ }^{2+}$ ion (purple globe), the C-terminal residues from the neighboring monomer (light green), the hydrocarbon tails (cyan), water molecules (red spheres), and sulfur atom of the ligand (orange). (D) Interactions between the ligands and the key catalytic residues in $\Delta z$ FPS-MDI and the side chains of E141 and H103 from $\Delta z$ FPS-APO (pink sticks). (E) Fo-Fc OMIT maps calculated for the ligand(s) bound in $\Delta z$ FPS-MI (middle) and $\Delta z$ FPS-MDI (right). Both were contoured at the 2.0 level.

successive isoprene units. ${ }^{18}$ The ligand in the $\mathrm{S} 1$ site interacts with the side chains of N88, R89, R90, Y103, L106, and R137. Among these, L106, which regulates the product chain length in $z$ FPS, ${ }^{17}$ is also crucial for ligand positioning. Two alternative binding modes potentially representing the transient binding states of the $\beta$-phosphate group from the S2 ligand were observed (Figure 1C), which may be regulated by the side chains of S131, R249, R255, and S257. The side chain from $\mathrm{N} 134$ interacts with both the $\alpha$-phosphate group and the allylic tail from the S2 ligand.

The $\Delta z$ FPS-MI and $\Delta z$ FPS-MDI structures suggest that $\mathrm{N} 134$ can serve as a general base for proton removal from the $\mathrm{S} 2$ ligand (Figure 1D). The $\mathrm{N}$ atom and the $\mathrm{O}$ atom of the side chain from N134 are close to the diphosphate group and the $\mathrm{C} 2 / \mathrm{C} 4$ atom of the ligand, respectively. Nucleophilic attack at the $\mathrm{C} 1$ atom of the $\mathrm{S} 1$ ligand by the $\pi$-electrons from the S2 ligand results in condensation. In $\Delta z$ FPS-MDI, with a distance of $2.9 \AA$ between the $\mathrm{C} 4$ atom from the S2 ligand and the C1 atom from the S1 ligand, head-to-tail condensation occurs. For the head-to-middle condensation reaction, the distance between the $\mathrm{C} 2$ atom of the S2 ligand and the $\mathrm{C} 1$ atom of the S1 ligand is $4.9 \AA$. $\Delta z$ FPS-MI shows an even longer distance. The ligand positioning seems to be more favored for the head-to-tail condensation by the short reacting distance. The occurrence of the head-to-middle condensation function may need a greater potential in the ionization of the S1 ligand.

The catalytic mechanism of head-to-middle condensation was proposed previously in a lavandulyl diphosphate synthase ${ }^{24}$
(LPPS, PDB ID: 5HC6), a cis-PTS with 39\% amino acid sequence identity to $z$ FPS. Superposition of LPPS and $\Delta z$ FPSMDI showed a highly conserved active site, with most substrate-binding residues identical (Figure S6A). The corresponding position of $z$ FPS-H103 is LPPS-H93. Two catalytic residues in the S1 site are different: $z$ FPS-R90/LPPSL80 and $z$ FPS-N88/LPPS-H78 (Scheme 1). According to our complex structures, $z$ FPS-R90 potentially regulates the opening/closing of S1 site (Figure 1B,C). LPPS-L80 may play a similar role. On the other hand, as proposed previously, LPPS$\mathrm{H} 78$ is the key catalytic residue for the head-to-middle reaction in LPPS, facilitating the diphosphate release from the S1 ligand. ${ }^{24}$ However, in $\Delta z$ FPS, the corresponding position of LPPS-H78 is $\Delta z$ FPS-N88. Alternatively, the Y103 residue of the $\Delta z$ FPS-H103Y mutant can play a crucial role in the ionization reaction of the diphosphate group (Scheme 1). In contrast, $\mathrm{H} 103$ in the $\Delta z$ FPS structure $(\Delta z \mathrm{FPS}$-APO) forms a hydrogen bond with E141, which inhibits the bonding between H103 and the diphosphate group from the S1 ligand (Figure 1D). The head-to-middle reaction is much less favored under this situation. As the enzymatic assays showed, $\Delta z$ FPS catalyzed head-to-middle condensation only in the absence of IPP (Figure 2). The elimination function served by Y103 in $\Delta z$ FPS-H103Y may also contribute to the small amounts of limonene found in the product profile. Revealed by structural comparison, the residue served for the diphosphate elimination in di-TPS Rv3378c is Y51 ${ }^{21}$ and the corresponding position is close to Y103 in $\Delta z$ FPS-MI (Figure S7). 


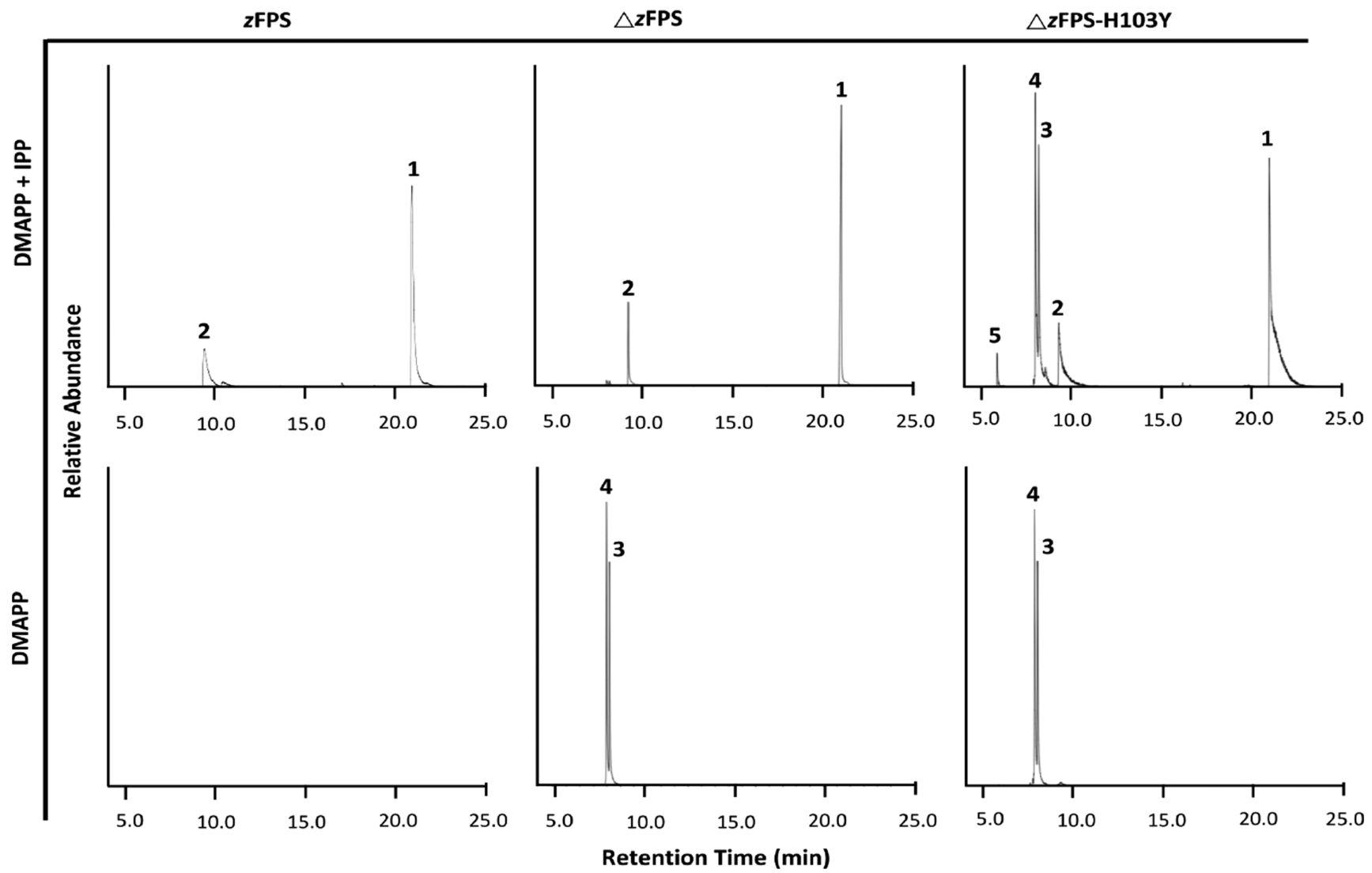

Figure 2. GC chromatograms of the enzyme products from $z$ FPS (without the signal peptide), $\Delta z$ FPS, and mutant $\Delta z$ FPS-H103Y. The peaks are identified as follows: 1, Z,Z-farnesol; 2, nerol; 3, unidentified irregular product; 4, lavandulol; and 5, limonene. RT, retention time (in minutes).

Scheme 1. Catalytic Mechanisms of $\Delta z$ FPS

A

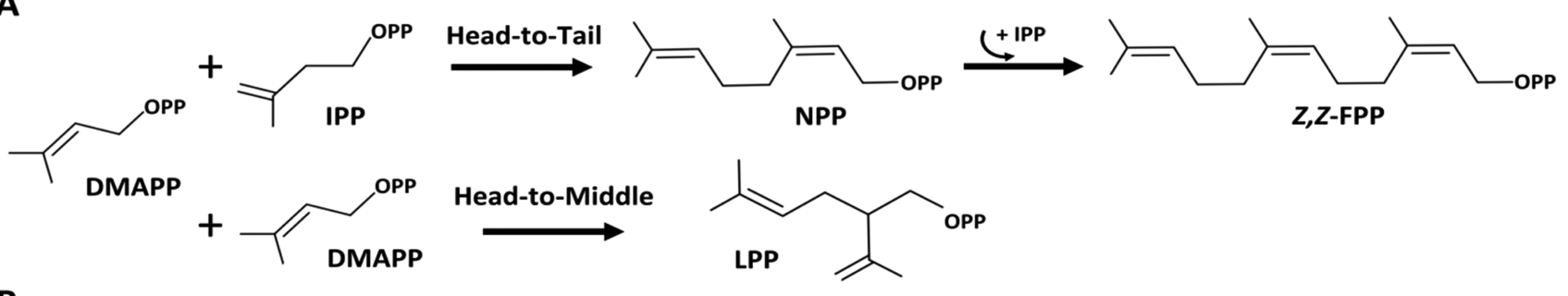

B

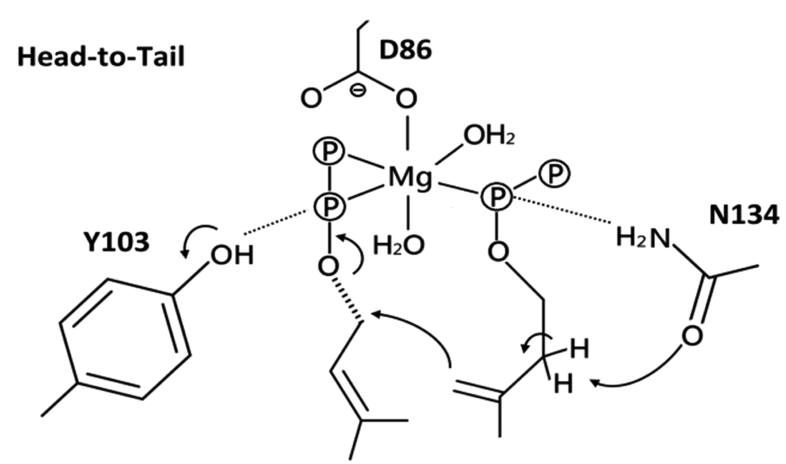

The head-to-tail condensation mechanism in cis-PTS, as proposed previously, ${ }^{18}$ starts with the binding of an allylic ligand in the S1 site. The homoallylic ligand then binds to the S2 site in the form of a MgIPP complex for the successive reaction. However, from the $\Delta z$ FPS-MD complex structure, only the S2 site was occupied by one DMSPP molecule, which may represent the starting configuration for head-to-middle

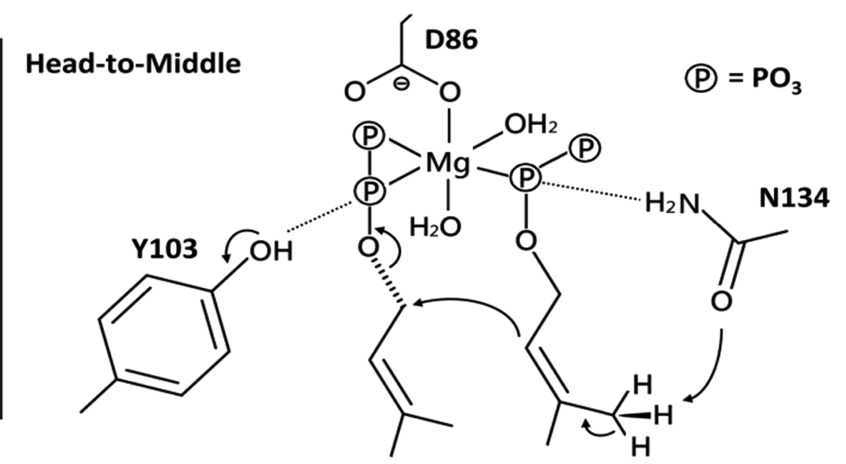

catalysis. This binding was initially assisted by the side chains of E133 and Y266, and C-terminal R296 from the neighboring monomer (Figure S5C). A second DMAPP molecule accompanied by $\mathrm{Mg}^{2+}$ then moves into the $\mathrm{S} 1$ site.

The adverse turn of the binding order for the head-to-middle reaction may be attributed to the S1 ligand (Figure S8), in which ready ionization of the diphosphate group is crucial. The 
reactive nature of the S1 ligand would result in an unstable binding; thus, the binding sequence is opposite compared to that in the head-to-tail reaction. We suppose that both of the binding sequences for head-to-tail and head-to-middle condensation reactions can be adopted in $\Delta z$ FPS.

The final coverage of the catalytic pocket is accomplished by the extended $\mathrm{C}$-terminal segment from the neighboring monomer (Figure 1B). The backbone of G299 on the terminus stabilizes the S2 ligand, and the side chain of R297 binds to two water molecules of the $\mathrm{Mg}^{2+}$-coordinated complex. The $\mathrm{Mg}^{2+}$ ion will eventually migrate to the diphosphate group of the $\mathrm{S} 1$ ligand and then leave as a MgPPi complex. ${ }^{18}$ The elongated product then occupies the $\mathrm{S} 1$ site for the subsequent reaction. The C-terminal segment is crucial because the $\Delta z$ FPS-H103Y/ R297A mutant was inactivated, a tremendous loss of activity in $\Delta z$ FPS-H103Y/R296A was also observed (Figure S3E,F).

Most PTSs reported to date are classified with a specific functional mechanism. However, a cis-PTS from Methanosarcina acetivorans was identified that naturally synthesizes both head-to-tail and head-to-middle reaction products. ${ }^{25}$ Using a structural approach, the potential of the multifunctional character of $\Delta z$ FPS was brought to light. The 103 position in $z$ FPS is not the only factor to regulate the functional specificity. With the $\mathrm{N}$-terminal segment retained, the enzymatic assay showed no irregular products in $z$ FPS (Figure 2). On the basis of the crystal structures of $\Delta z$ FPS-MI and $\Delta z$ FPS-MDI, the 23residue $\mathrm{N}$-terminus might be able to extend to the active site of the neighboring monomer near the C-terminus, and this $\mathrm{N}$ terminal segment is one of the important functional regulators of $z$ FPS, apparently inhibiting the occurrence of the head-tomiddle condensation reaction. However, traceable amounts of the irregular products can still be detected when DMAPP was provided in excessive concentration (Figure S3B). The reason why $z$ FPS could not be crystallized without the $\mathrm{N}$-terminal truncation is likely the flexibility of this segment, which probably restricts the binding of IPP in the S2 site.

\section{CONCLUSIONS}

In the present study, the head-to-middle condensation function was observed in the $\mathrm{N}$-terminal truncated $\Delta z \mathrm{FPS}$. High substrate specificity was demonstrated in this enzyme as the irregular function only occurs without the presence of IPP. The regulatory nature of this enzyme was characterized on the basis of the structural information obtained from complex structures accompanied by mutagenesis and enzymatic assays. Catalytic mechanisms of both the typical head-to-tail and irregular headto-middle condensation reactions were proposed. The regulatory role played by the residue at positon 103 in $z$ FPS was elucidated as well. These findings contribute to furthering our understanding about the formation of highly diverse prenyl phytochemicals.

\section{METHODS}

Protein Expression and Purification. The nucleotide sequence of $\Delta z$ FPS from tomato lacking the $\mathrm{N}$-terminal segment (residues 1-70) with the double mutations D71M and E75A was synthesized on a codon-optimized basis of the Escherichia coli translational system (http://www.genscript. com). As the native hydrophilic N-terminal is flexible, which may have a negative effect on crystallization, we had mutated the two residues to hydrophobic methionine and alanine to improve the crystal packing. The obtained nucleotides were then cloned into the pET-32 Xa/LIC vector (www. merckmillipore.com), which includes a Factor Xa cleavage site between the upstream His-tag and the downstream coding sequence. The same expression strategy was applied to the mutants in this study. The mutants obtained from site-directed mutagenesis were produced using PCR-based methods by the following primers $\left(3^{\prime}-5^{\prime}\right): \Delta z$ FPS-H103Y, forward primer: CGTTTCGGAAGGTTATAAACACCTG; reverse primer: CAGGTGTTTATAACCTTCCGAAACG. $\Delta z$ FPS-H103Y/ R2 $96 \mathrm{~A}$, forw a rd primer: G CA G C G T CATGCTCGTTTTGGTGGTCACACG; reverse primer: CGTGTGACCACCAAAACGAGCATGACGCTGC. $\Delta z$ FPSH 103 Y/R297A, forward primer: GCAGCGTCATCGTGCTTTTGGTGGTCACACG; reverse primer: CGTGTGACCACCAAAAGCACGATGACGCTGC. All PCR reactions were performed using high-fidelity DNA polymerase KOD-Plus following the manufacturer's instructions (www. toyobo-global.com). The obtained PCR products were then digested by DpnI (www.neb.com) at $37{ }^{\circ} \mathrm{C}$ for $1 \mathrm{~h}$ and transformed into $E$. coli $\mathrm{DH} 5 \alpha$ cells for plasmid production.

Each constructed clone was transformed into E. coli BL21 (DE3) cells for protein expression. The cells were grown to A600 of 0.6 at $37^{\circ} \mathrm{C}$ in $\mathrm{LB}$ medium containing $50 \mu \mathrm{g} \mathrm{mL}^{-1}$ of ampicillin, induced with $0.5 \mathrm{mM}$ isopropyl-1-thio- $\beta$-D-galactopyranoside, and grown for $16 \mathrm{~h}$ at $16^{\circ} \mathrm{C}$. They were then harvested by centrifugation at $6000 \mathrm{~g}$ at $4{ }^{\circ} \mathrm{C}$, resuspended in 50

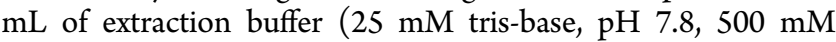
$\mathrm{NaCl}, 10 \mathrm{mM}$ imidazole), and broken by a French Press (Constant Systems Ltd). For protein purification, the lysates were recovered by centrifugation at $20000 \mathrm{~g}$ for $40 \mathrm{~min}$ at $4{ }^{\circ} \mathrm{C}$. $\Delta z$ FPS or $\Delta z$ FPS mutants with $\mathrm{N}$-terminal thioredoxin and His-tag fusion in supernatants were then applied to binding columns with nickel-nitrilotriacetic acid (Ni-NTA) agarose resin (https://www.qiagen.com). The imidazole concentration of the wash buffer was increased to $40 \mathrm{mM}$, and the recombinant protein fractions were eluted at an imidazole concentration between 50 and $70 \mathrm{mM}$. After processing by Factor Xa protease digestion to remove the N-terminal fusion tags (www.neb.com), the mixtures were then passed through a second Ni-NTA column to remove the uncleaved fusion protein and free fusion tags. The purified protein was concentrated to $10 \mathrm{mg} \mathrm{mL} L^{-1}$ in a buffer solution of $25 \mathrm{mM}$ tris-base, $\mathrm{pH} 8.0,150 \mathrm{mM} \mathrm{NaCl}$. SDS-polyacrylamide gel electrophoresis analyses were performed to demonstrate the protein purity. The purified proteins were frozen with liquid nitrogen and stored at $-80{ }^{\circ} \mathrm{C}$.

Crystallization and Data Collection. Crystals of $\Delta z \mathrm{FPS}$ $(\Delta z$ FPS-APO) and $\Delta z$ FPS-H103Y $(\Delta z$ FPS-M $)$ were grown by mixing $2 \mu \mathrm{L}$ of protein with $2 \mu \mathrm{L}$ of reservoir solution using the sitting-drop vapor diffusion method at $18{ }^{\circ} \mathrm{C}$. The $\Delta z$ FPS crystals were obtained in a reservoir solution of 70\% 2-methyl2,4-pentanediol (MPD), $0.1 \mathrm{M}$ Tris, $\mathrm{pH}$ 8.0. The $\Delta z$ FPS$\mathrm{H} 103 \mathrm{Y}$ mutant was crystallized in a reservoir solution of $5 \%$ (w/v) PEG 8000, 40\% MPD, $0.1 \mathrm{M}$ cacodylate, $\mathrm{pH}$ 7.0. Complex crystals of $\Delta z$ FPS-MI $\left(\Delta z\right.$ FPS-H103Y $\left./ \mathrm{Mg}^{2+} / \mathrm{IPP}\right)$, $\Delta z$ FPS-MD $(\Delta z$ FPS-H103Y/DMSPP $)$, and $\Delta z$ FPS-MDI $\left(\Delta z\right.$ FPS-H103Y $\left./ \mathrm{Mg}^{2+} / \mathrm{DMSPP} / \mathrm{IPP}\right)$ were obtained with reservoir solution of $13 \%$ PEG10k, $20 \mathrm{mM} 18$-crown- $6,{ }^{26} 0.1$ $\mathrm{M}$ MES, $\mathrm{pH}$ 6.5. All diffraction data were collected using X-rays of wavelength $1.000 \AA$, recorded at cryogenic temperatures with a cryoprotectant solution of $20-25 \%$ glycerol. Data of $\Delta z$ FPS APO were collected at the beamline BL5-A of the Photon Factory in Japan, with an ADSC Quantum 315r CCD detector. 
Data for the apo-form $\Delta z$ FPS-H103Y crystal were collected at the beamline BL12B2 of SPring-8 in Japan, using an ADSC Quantum 210 CCD detector. Data for other complex crystals including $\Delta z$ FPS-MI and $\Delta z$ FPS-MD were collected using the beamline BL15A1 of the National Synchrotron Radiation Research Center (NSRRC) in Taiwan. The $\Delta z$ FPS-MDI complex data was collected at the NSRRC Taiwan Photon Source (TPS) beamline TPS-05A with a Rayonix MX300-HS CCD detector. All diffraction data were processed and scaled using the HKL2000 program. ${ }^{27}$

Structure Determination and Refinement. All crystal structures in this study were determined by molecular replacement using the MOLREP program of the CCP4 program suite. ${ }^{28}$ The E. coli UPPS (PDB code: 1JP3) was used as the initial search model. Both $\Delta z$ FPS and $\Delta z$ FPS$\mathrm{H} 103 \mathrm{Y}$ crystals belong to space group $\mathrm{P}_{3} 2_{1} 2$, with one protein molecule in an asymmetric unit. The complex structures, $\Delta z$ FPS-MDI, $\Delta z$ FPS-MI, and $\Delta z$ FPS-MD, were crystallized in space group $P 2_{1} 2_{1} 2_{1}$, with two molecules per asymmetric unit. Throughout the refinement, $5 \%$ of randomly selected data were set aside for cross-validation with Rfree values. Manual modifications of the models were performed using the program Coot. $^{29}$ Difference Fourier (Fo-Fc) maps were calculated to locate the solvent molecules. All crystal structures were refined using Refmac5, ${ }^{30}$ including individual isotropic B-factor refinement. The molecular figures were produced using UCSF Chimera. ${ }^{31}$

In Vitro Enzymatic Assays. Enzymatic assays were

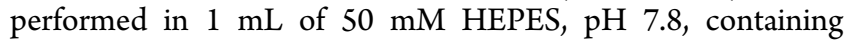
$100 \mathrm{mM} \mathrm{KCl}, 7.5 \mathrm{mM} \mathrm{MgCl} 2,5 \%$ (w/v) glycerol, and $5 \mathrm{mM}$ DTT. For each assay, $50 \mu \mathrm{g}$ of zFPS, $\Delta z$ FPS or $\Delta z$ FPS mutants was incubated at $32{ }^{\circ} \mathrm{C}$ for $2 \mathrm{~h}$, with different combinations of DMAPP, IPP, neryl diphosphate (NPP), and GPP (www. echelon-inc.com) at $65 \mu \mathrm{M}$. The reaction products were then dephosphorylated by adding 20 units of alkaline phosphatase (www.neb.com) and incubated at $37{ }^{\circ} \mathrm{C}$ for $1 \mathrm{~h}$. The final products were extracted three times, each by vortexing with 2 $\mathrm{mL}$ of pentane. After separating the water phase by centrifugation, the obtained organic phase was then concentrated to $10 \mu \mathrm{L}$. The products were analyzed on an Agilent 5975C Series GC/MSD. The samples in pentane were injected directly into the GC-MS system. Separation was assured with a DB-5 capillary column (J\&W Scientific; $30 \mathrm{~m}, 0.25 \mathrm{~mm}$ inside diameter). The temperature program used was isothermal 70 ${ }^{\circ} \mathrm{C}$ for $2 \mathrm{~min}$, followed by a linear gradient $\left(8^{\circ} \mathrm{C} \mathrm{min}^{-1}\right)$ to 250 ${ }^{\circ} \mathrm{C}$. Mass spectra from NIST Mass Spectral Library and authentic standards were used for product confirmation. Authentic standards including lavandulol, farnesol, limonene, and $\alpha$-terpineol were purchased from Sigma (www. sigmaaldrich.com), and Z,Z-farnesol was purchased from Echelon (www.echelon-inc.com).

Analytical Ultracentrifugation. The SV experiments were performed at $50000 \mathrm{rpm}$ using a 4-hole AnTi60 rotor at $20^{\circ} \mathrm{C}$ in a Beckman Optima XL-I AUC equipped with absorbance optics. The purified $\Delta z$ FPS samples were collected and diluted to different concentrations: $1,0.5$, and $0.1 \mathrm{mg} \mathrm{mL}^{-1}$, in a protein storage buffer $(25 \mathrm{mM}$ tris-base, $150 \mathrm{mM} \mathrm{NaCl}, 1 \mathrm{mM}$ DTT, pH 8.0). Standard $12 \mathrm{~mm}$ aluminum double-sector centerpieces were filled with protein solution, with a reference cell containing the blank buffer. Quartz windows were used with absorbance optics (OD 280) in a continuous mode without averaging. No time interval was set between scans. The data were analyzed with a $c(s)$ distribution of the Lamm equation solutions calculated using SEDFIT Version 14.1 (http://analyticalultracentrifugation.com). Software Sednterp (http://www.jphilo.mailway.com) was used to estimate the protein partial specific volume (Vbar), buffer density (1.0051 g $\left.\mathrm{mL}^{-1}\right)$, and buffer viscosity $(0.0102331 \mathrm{P})$ at $20^{\circ} \mathrm{C}$.

\section{ASSOCIATED CONTENT}

\section{Supporting Information}

The Supporting Information is available free of charge on the ACS Publications website at DOI: 10.1021/acsomega.6b00562.

Experimental materials, methods, supporting tables, and figures (PDF)

Crystallographic information file for $5 \mathrm{HXN}$ (CIF)

Crystallographic information file for $5 \mathrm{HXO}$ (CIF)

Crystallographic information file for 5HXP (CIF)

Crystallographic information file for 5HXQ (CIF)

Crystallographic information file for 5HXT (CIF)

\section{AUTHOR INFORMATION}

\section{Corresponding Authors}

*E-mail: chengung@gate.sinica.edu.tw. Tel: +886-2-2785-5696 (C.-C.L.).

*E-mail: ahjwang@gate.sinica.edu.tw. Tel: +886-2-2788-1981. Fax: +886-2-2788-2043 (A.H.-J.W.).

ORCID

Yueh-Te Chan: 0000-0002-7759-5511

Funding

This work was supported by grants from the Taiwan Protein Project (MOST105-0210-01-12-01 and MOST106-0210-0115-04), the Academia Sinica-Industrial Technology Research Institute Collaboration Project (23yy-y110505), and Academia Sinica and Ministry of Science and Technology (MOST1060210-01-15-02).

\section{Notes}

The authors declare no competing financial interest.

\section{ACKNOWLEDGMENTS}

We thank many groups at Academia Sinica: Yu-Ching Wu from the Small Molecule Metabolomics Core Lab, Institute of Plant and Microbial Biology, for use of GC-MS instrument; HuiLing Shih from Core Facilities for protein structural analysis, Institute of Biological Chemistry, for crystallization screening processing; and Meng- $\mathrm{Ru} \mathrm{Ho}$ from the Biophysics Core Facility, Scientific Instrument Center, for AUC analysis. We are also grateful to the National Synchrotron Radiation Research Center (NSRRC) of Taiwan for the synchrotron beam time allocations.

\section{REFERENCES}

(1) Pichersky, E.; Gang, D. R. Trends Plant Sci. 2000, 5, 439-445.

(2) Gershenzon, J.; Dudareva, N. Nat. Chem. Biol. 2007, 3, 408-414.

(3) Christianson, D. W. Chem. Rev. 2006, 106, 3412-3442.

(4) Bohlmann, J.; Meyer-Gauen, G.; Croteau, R. Proc. Natl. Acad. Sci. U.S.A. 1998, 95, 4126-4133.

(5) Coates, R. M.; Denissen, J. F.; Juvik, J. A.; Babka, B. A. J. Org. Chem. 1988, 53, 2186-2192.

(6) Kasahara, H.; Hanada, A.; Kuzuyama, T.; Takagi, M.; Kamiya, Y.; Yamaguchi, S. J. Biol. Chem. 2002, 277, 45188-45194.

(7) Rohmer, M.; Knani, M.; Simonin, P.; Sutter, B.; Sahm, H. Biochem. J. 1993, 295, 517-524.

(8) Charon, L.; Hoeffler, J. F.; Pale-Grosdemange, C.; Lois, L. M.; Campos, N.; Boronat, A.; Rohmer, M. Biochem. J. 2000, 346, 737-742. 
(9) Seemann, M.; Bui, B. T. S.; Wolff, M.; Miginiac-Maslow, M.; Rohmer, M. FEBS Lett. 2006, 580, 1547-1552.

(10) Liang, P. H.; Ko, T. P.; Wang, A. H. J. Eur. J. Biochem. 2002, 269, 3339-3354.

(11) Vandermoten, S.; Haubruge, É.; Cusson, M. Cell. Mol. Life Sci. 2009, 66, 3685-3695.

(12) Liu, Z.; Zhou, J.; Wu, R.; Xu, J. J. J. Chem. Theory Comput. 2014, 10, 5057-5067.

(13) Köksal, M.; Jin, Y.; Coates, R. M.; Croteau, R.; Chris-tianson, D. W. Nature 2011, 469, 116-120.

(14) Ko, T. P.; Chen, Y. K.; Robinson, H.; Tsai, P. C.; Gao, Y. G.; Chen, A. P. C.; Wang, A. H. J.; Liang, P. H. J. Biol. Chem. 2001, 276, 47474-47482.

(15) Fujihashi, M.; Zhang, Y. W.; Higuchi, Y.; Li, X. Y.; Koyama, T.; Miki, K. Proc. Natl. Acad. Sci. U.S.A. 2001, 98, 4337-4342.

(16) Sallaud, C.; Rontein, D.; Onillon, S.; Jabés, F.; Duffé, P.; Giacalone, C.; Thoraval, S.; Escoffier, C.; Herbette, G.; Leonhardt, N.; Causse, M.; Tissier, A. Plant Cell. 2009, 21, 301-317.

(17) Kang, J. H.; Gonzales-Vigil, E.; Matsuba, Y.; Pichersky, E.; Barry, C. S. Plant Physiol. 2014, 164, 80-91.

(18) Guo, R. T.; Ko, T. P.; Chen, A. P. C.; Kuo, C. J.; Wang, A. H. J.; Liang, P. H. J. Biol. Chem. 2005, 280, 20762-20774.

(19) Lesburg, C. A.; Zhai, G.; Cane, D. E.; Christianson, D. W. Science 1997, 277, 1820-1824.

(20) Whittington, D. A.; Wise, M. L.; Urbansky, M.; Coates, R. M.; Croteau, R. B.; Christianson, D. W. Proc. Natl. Acad. Sci. U.S.A. 2002, 99, 15375-15380.

(21) Chan, H. C.; Feng, X.; Ko, T. P.; Huang, C. H.; Hu, Y.; Zheng, Y.; Bogue, S.; Nakano, C.; Hoshino, T.; Zhang, L.; Lv, P.; Liu, W.; Crick, D. C.; Liang, P. H.; Wang, A. H. J.; Oldfield, E.; Guo, R. T. J. Am. Chem. Soc. 2014, 136, 2892-2896.

(22) Ozaki, T.; Zhao, P.; Shinada, T.; Nishiyama, M.; Kuzuyama, T. J. Am. Chem. Soc. 2014, 136, 4837-4840.

(23) Demissie, Z. A.; Erland, L. A. E.; Rheault, M. R.; Mahmoud, S. S. J. Biol. Chem. 2013, 288, 6333-6341.

(24) Liu, M.; Chen, C. C.; Chen, L.; Xiao, X.; Zheng, Y.; Huang, J. W.; Liu, W.; Ko, T. P.; Cheng, Y. S.; Feng, X.; Oldfield, E.; Guo, R. T.; Ma, Y. Angew. Chem. 2016, 128, 4799-4802.

(25) Ogawa, T.; Emi, K. I.; Koga, K.; Yoshimura, T.; Hemmi, H. FEBS J. 2016, 283, 2369-2383.

(26) Lee, C. C.; Maestre-Reyna, M.; Hsu, K. C.; Wang, H. C.; Liu, C. I.; Jeng, W. Y.; Lin, L. L.; Wood, R.; Chou, C. C.; Yang, J. M.; Wang, A. H. J. Angew. Chem., Int. Ed. 2014, 53, 13054-13058.

(27) Otwinowski, Z.; Minor, W. Methods Enzymol. 1997, 276, 307326.

(28) Bailey, S. Acta Crystallogr., Sect. D: Biol. Crystallogr. 1994, 50, $760-763$.

(29) Emsley, P.; Cowtan, K. Acta Crystallogr., Sect. D: Biol. Crystallogr. 2004, 60, 2126-2132.

(30) Murshudov, G. N.; Vagin, A. A.; Dodson, E. J. Acta Crystallogr., Sect. D: Biol. Crystallogr. 1997, 53, 240-255.

(31) Pettersen, E. F.; Goddard, T. D.; Huang, C. C.; Couch, G. S.; Greenblatt, D. M.; Meng, E. C.; Ferrin, T. E. J. Comput. Chem. 2004, $25,1605-1612$. 